\title{
Bioinformatics analysis of differentially expressed genes in subchondral bone in early experimental osteoarthritis using microarray data
}

\author{
Zhao Wang ${ }^{1}$, Yong $\mathrm{Ji}^{2^{*}}$ and Hong-wei Bao ${ }^{1}$
}

\begin{abstract}
Background: Osteoarthritis $(\mathrm{OA})$ is the most common arthritic disease in humans, affecting the majority of individuals over 65 years of age. The aim of this study is to identify the gene expression profile specific to subchondral bone in OA by comparing the different expression profiles in experimental and sham-operation groups.

Methods: Gene expression profile GSE30322 was downloaded from the Gene Expression Omnibus (GEO) database. Differentially expressed genes (DEGs) were obtained by limma package. And Database for Annotation, Visualization and Integrated Discovery (DAVID) databases were further used to identify the potential gene ontology (GO) and Kyoto Encyclopedia of Genes and Genomes (KEGG) pathways. Furthermore, a protein-protein interaction (PPI) network was constructed and significant modules were extracted.

Results: Totally, 588 DEGs were identified including 199 upregulated DEGs and 389 downregulated DEGs screened in $\mathrm{OA}$ and sham-operation. GO showed that DEGs were significantly enhanced for ribosomal subunit export from nucleus and molting cycle. KEGG pathway analysis revealed that target genes were enriched in thiamine metabolism.

Conclusion: These key candidate DEGs that affect the progression of $\mathrm{OA}$, and these genes might serve as potential therapeutic targets for OA.
\end{abstract}

Keywords: Osteoarthritis, Differentially expressed genes, Bioinformatics analysis, Gene ontology

\section{Introduction}

Osteoarthritis $(\mathrm{OA})$ is a degenerative disease characterized by the gradual degeneration of articular cartilage, joint stiffness, and loss of function [1]. It was reported that over 27 million adults are affected by $\mathrm{OA}$ in the USA [2]. OA is a complex pathophysiological process involving inflammation, subchondral bone modification, and osteophyte formation. Subchondral bone alteration present to the cartilage degeneration and thus more

\footnotetext{
* Correspondence: 42394176@qq.com

${ }^{2}$ Department of General Surgery, Jingjiang People's Hospital, No. 28, Zhongzhou Road, Jingjiang, Taizhou 214500, Jiangsu Province, China Full list of author information is available at the end of the article
}

studies should be focused on the subchondral bone alteration.

Subchondral bone consists tripartite: subchondral bone plate, trabecular bone, and bone marrow space [3]. It has been stated that most of the OA patients accompanied by the alterations of the subchondral bone [4]. Subchondral bone could transport nutrients or cytokines to the overlying cartilage. Meanwhile, subchondral bone cells contacted with chondrocyte and thus influence cartilage metabolism. A better understanding of the early molecular mechanism changes of subchondral bone in vivo may contribute to elucidating the pathogenesis of OA. Therefore, it is crucial to explore the differentially 
expressed genes (DEGs) in vivo and thus we could revealed new targets for OA [5].

Microarray technology has been used to obtain information on the genetic alteration that occurs during many diseases [6, 7]. Here, we downloaded the gene expression profile GSE30322 from the Gene Expression Omnibus database (GEO), including gene expression data for subchondral bone samples from five medial meniscectomy and medial collateral ligament transection group and five sham-operated group. Based upon this research, identifying DEGs and enriching their functions and signaling pathways may help reveal potential targets of early OA.

\section{Materials and methods}

\section{Gene expression microarray data}

The gene expression profile GSE30322 (https://www. ncbi.nlm.nih.gov/geo/query/acc.cgi?acc=GSE30322) was downloaded from the Gene Expression Omnibus (GEO, www.ncbi.nlm.nih.gov/geo/). GSE30322 was based on Agilent-014879 Whole Rat Genome Microarray 4x44K G4131F (Probe Name version) platform. GSE30322 dataset contained ten samples, including five bone 4 weeks post-surgery samples (E-group), and five sham-operated group (S-group) 4 weeks post-surgery samples.

\section{DEGs in E-group and intact S-group samples}

The raw data files were downloaded and then python scripts for matrix transformation were used. The analysis was carried out using Limma package from Bioconductor project. In this study, genes with $P<.05$ and [log fold change (FC)] $>2$ were defined as DEGs. The DEGs data were then processed by $\mathrm{R}$ software (pheatmap package) to draw a heatmap and volcano plot.

\section{GO and KEGG analysis of DEGs}

Target genes list were submitted to the DAVID 6.8 (https://david.ncifcrf.gov/) to analyze candidate DEG functions and Kyoto Encyclopedia of Genes and Genomes (KEGG) of the overlapping genes. DEG functions, also named as Gene ontology (GO), mainly including biological process (BP), molecular function (MF), and cellular component (CC). $P$ value less than 0.05 was considered as cut-off criterion [8-10].

\section{Protein-protein interaction (PPI)}

We used the online database STRING (Search Tool for the Retrieval of Interacting Genes, https://string-db.org/) to better illustrate the potential interactive relationships among the DEGs [11]. Then, the Cytoscape software was utilized for analyzing the interactions with a combined score $>0.4$ (http://www.cytoscape.org/). Finally, the plugin Molecular Complex Detection (MCODE) was used to filter the significant modules from the PPI network for the
Table 1 The top 10 upregulated DEGs in early experimental osteoarthritis with $P$ value $<0.05$

\begin{tabular}{lll}
\hline Genesymbol & $\operatorname{logFC}$ & $P$ value \\
\hline Rhox5 & 2.271332 & 0.008302 \\
Bex1 & 2.035975 & 0.040834 \\
RGD1309085 & 1.726136 & 0.012291 \\
Nsg1 & 1.658621 & 0.001075 \\
Klhdc5 & 1.63377 & 0.007725 \\
Trpc4 & 1.609058 & 0.003723 \\
Klrd1 & 1.606762 & 0.00939 \\
Fgfbp3 & 1.604729 & 0.000907 \\
Gzma & 1.588101 & 0.011649 \\
Nr4a3 & 1.550933 & $1.16 \mathrm{E}-05$ \\
\hline
\end{tabular}

selection of hub genes (degree cut-off $=2$, node score cutoff $=0.2, k$-core $=2$, and max. depth $=100)$ [12].

\section{Results}

\section{Identification of DEGs}

After analyzing, differentially expression gene profiles were obtained. Totally, 588 DEGs were identified including 199 upregulated DEGs and 389 downregulated DEGs screened in OA and sham-operation. Top 10 up-DEGs and down-DEGs were listed in Table 1 and Table 2, respectively. A box plot of the sample data is provided in Fig. 1. Volcano plot of the different genes can be obtained in Fig. 2. Moreover, we provided heatmap of the top 50 different genes between E-group and S-group (Fig. 3).

\section{GO term enrichment analysis of DEGs}

Gene Ontology (GO) showed that up-DEGs were significantly enhanced for ribosomal subunit export from nucleus, ribosome localization, regulation of hemopoiesis, negative regulation of hemopoiesis, and rRNA-containing ribonucleoprotein complex export from nucleus. Downregulated DEGs were enriched for the molting cycle, hair cycle,

Table 2 The top 10 downregulated DEGs in early experimental osteoarthritis with $P$ value $<0.05$

\begin{tabular}{lll}
\hline Genesymbol & $\log \mathrm{FC}$ & $P$ value \\
\hline Ric8a & -4.51046 & $3.57 \mathrm{E}-06$ \\
Fth1 & -4.09365 & $2.18 \mathrm{E}-05$ \\
LOC305052 & -4.0216 & $1.25 \mathrm{E}-10$ \\
Pygl & -3.73589 & $3.01 \mathrm{E}-08$ \\
Cks2 & -3.61584 & $3.06 \mathrm{E}-07$ \\
Usp4 & -3.60799 & $2.21 \mathrm{E}-05$ \\
Rasl2-9 & -3.57891 & $1.54 \mathrm{E}-08$ \\
Cpox & -3.50355 & $3.86 \mathrm{E}-10$ \\
Rab7a & -3.43118 & $1.07 \mathrm{E}-08$ \\
Tmsb10 & -3.40738 & $1.24 \mathrm{E}-10$ \\
\hline
\end{tabular}




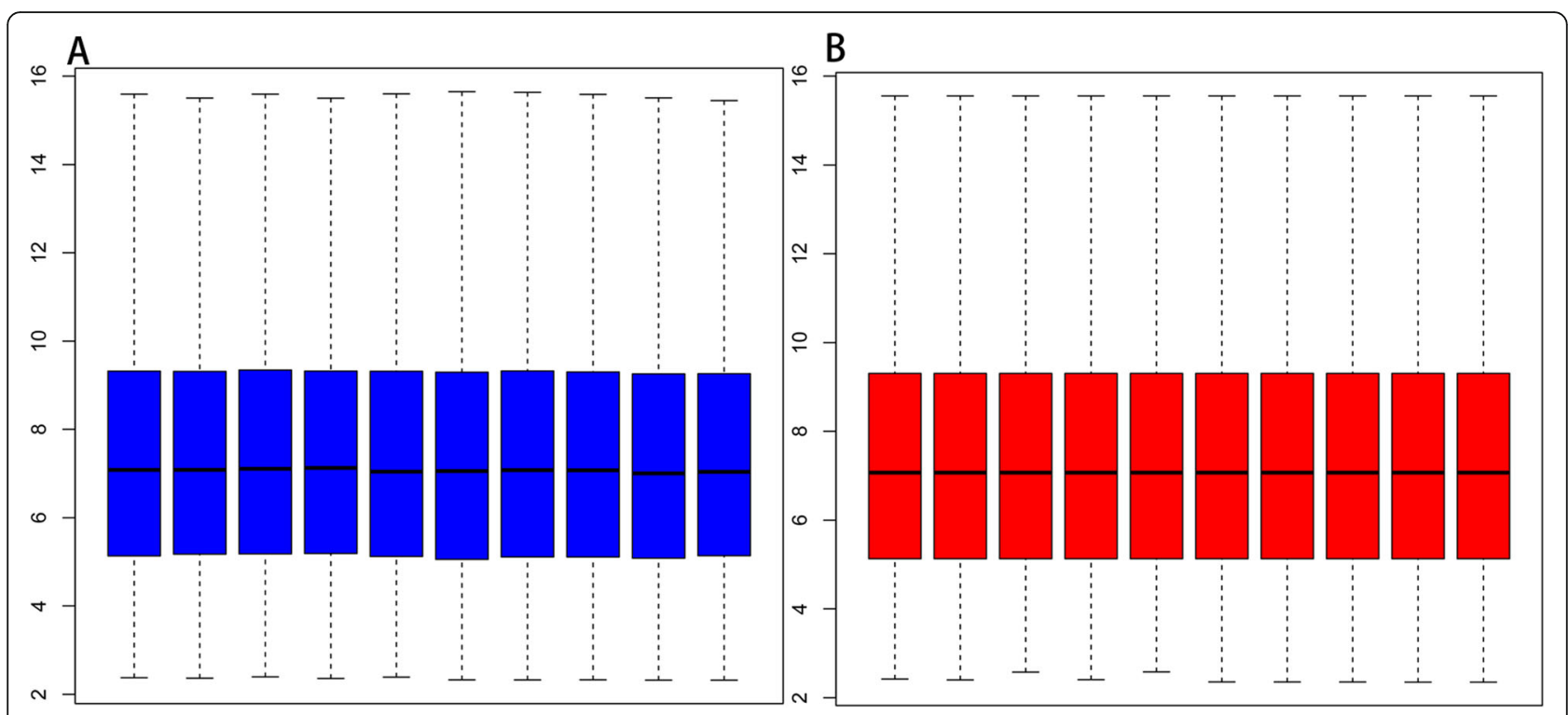

Fig. 1 Box plot for the sample data after normalization

molting cycle process, hair cycle process, and the skin epidermis development (Table 3 and Fig. 4).

\section{KEGG pathway analysis of DEGs}

The result of KEGG pathway analysis revealed that target genes were enriched in thiamine metabolism, regulation of lipolysis in adipocytes, central carbon metabolism in cancer, estrogen signaling pathway, collecting duct acid secretion, Rap1 signaling pathway, measles, sphingolipid metabolism, drug metabolismother enzymes, and circadian rhythm. These key pathways were showed in Table 4 and Fig. 5.

\section{Volcano}

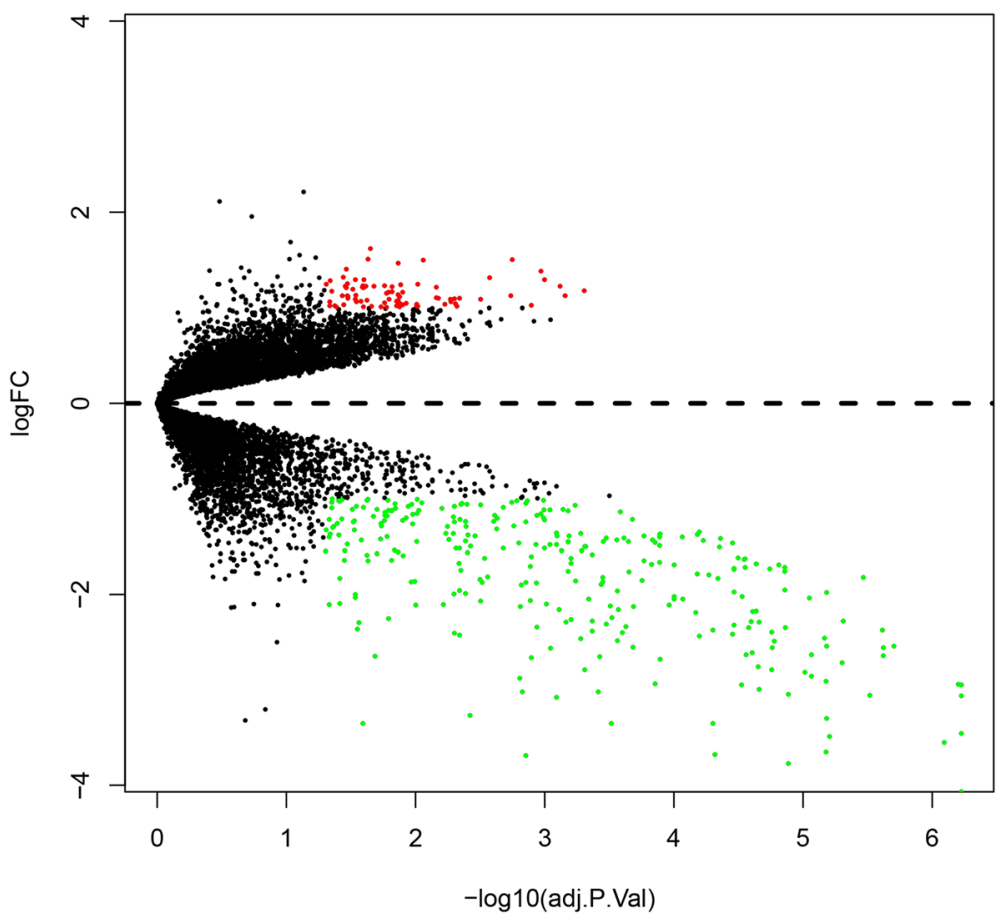

Fig. 2 Volcano plot of the different genes in E-group and S-group 


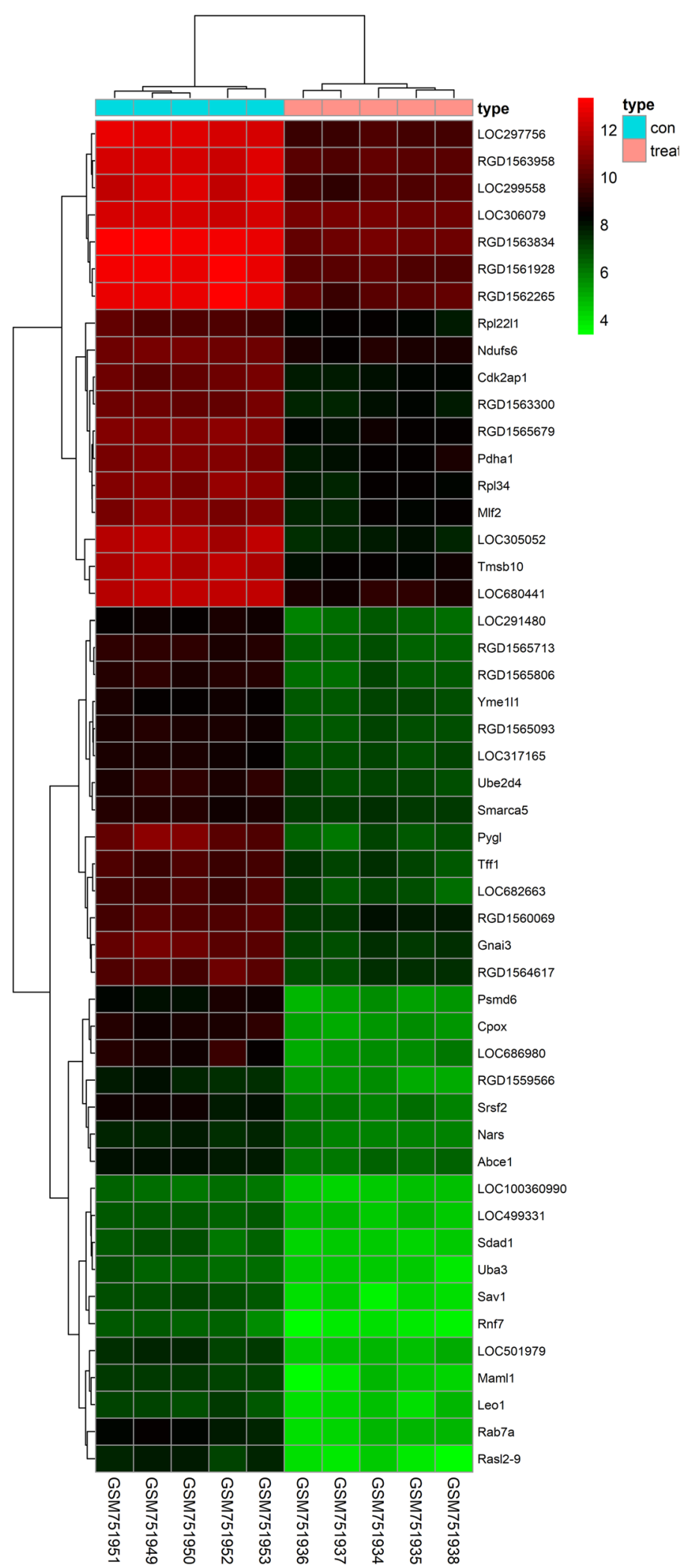

Fig. 3 Heat map of the top fifty different genes in E-group and S-group 
Table 3 Gene ontology analysis of differentially expressed genes in E-group and S-group according to BP, CC and MF

\begin{tabular}{|c|c|c|c|c|}
\hline ONTOLOGY & Description & $P$ value & $P$ adjust & gname \\
\hline $\mathrm{BP}$ & Ribosomal subunit export from nucleus & 0.000659 & 0.43601 & RASL2-9/SDAD1/ZFP593/RPS15 \\
\hline $\mathrm{BP}$ & Ribosome localization & 0.000659 & 0.43601 & RASL2-9/SDAD1/ZFP593/RPS15 \\
\hline $\mathrm{BP}$ & Regulation of hemopoiesis & 0.000698 & 0.43601 & $\begin{array}{l}\text { ADRM1/LEO1/CSF1/HMGB2/CALCA/RARA/ITPKB/NFE2L2/MEIS2/ERBB2/I } \\
\text { HH/GPR171/INHBA/TNFSF9/PPP2R3C/MIXL1/HIST2H4/GNAS/IL1F8/ZA } \\
\text { P70/RGD1309676 }\end{array}$ \\
\hline $\mathrm{BP}$ & Negative regulation of hemopoiesis & 0.001031 & 0.43601 & $\begin{array}{l}\text { LEO1/CALCA/RARA/ITPKB/NFE2L2/MEIS2/ERBB2/IHH/GPR171/MIXL1/ } \\
\text { HIST2H4 }\end{array}$ \\
\hline $\mathrm{BP}$ & $\begin{array}{l}\text { rRNA-containing ribonucleoprotein } \\
\text { complex export from nucleus }\end{array}$ & 0.001063 & 0.43601 & RASL2-9/SDAD1/ZFP593/RPS15 \\
\hline $\mathrm{BP}$ & Hair follicle development & 0.001079 & 0.43601 & SAV1/NANGL2/LHX2/GAL/INHBA/RBPJ/GNAS/LGR4/PRSS8 \\
\hline BP & Molting cycle & 0.001083 & 0.43601 & SAV1NANGL2/LHX2/GAL/INHBA/RBPJ/GNAS/LGR4/ARNTL/PRSS8 \\
\hline $\mathrm{BP}$ & Hair cycle & 0.001083 & 0.43601 & SAV1NANGL2/LHX2/GAL/INHBA/RBPJ/GNAS/LGR4/ARNTL/PRSS8 \\
\hline $\mathrm{BP}$ & Molting cycle process & 0.001245 & 0.43601 & SAV1/NANGL2/LHX2/GAL/INHBA/RBPJ/GNAS/LGR4/PRSS8 \\
\hline $\mathrm{BP}$ & Hair cycle process & 0.001245 & 0.43601 & SAV1NANGL2/LHX2/GAL/INHBA/RBPJ/GNAS/LGR4/PRSS8 \\
\hline CC & Cytosolic small ribosomal subunit & 0.000355 & 0.163784 & $\begin{array}{l}\text { LOC297756/RPS7/RPS26/RPS27A/RGD1565117/RGD1562381/RPS15/RG } \\
\text { D1559808 }\end{array}$ \\
\hline CC & Nuclear speck & 0.002082 & 0.276812 & $\begin{array}{l}\text { SURF2/EP400/SRSF2/PACSIN2/GLYR1/BASP1/KIF22/SNRPB2/MORF4L1/S } \\
\text { MC4/CKAP4/TCF3/NR3C1/HSPB3/HSPA1B/CDK12/CNOT7/EAF2 }\end{array}$ \\
\hline CC & Blood microparticle & 0.002809 & 0.276812 & ACTC1/CPN2/HBE1/PRSS1/HSPA1B/ACTG2/PROS1/HSPA1L/SERPINF2 \\
\hline CC & Small ribosomal subunit & 0.002991 & 0.276812 & $\begin{array}{l}\text { LOC297756/RPS7/RPS26/RPS27A/RGD1565117/RGD1562381/RPS15/RG } \\
\text { D1559808 }\end{array}$ \\
\hline CC & Cytosolic ribosome & 0.003002 & 0.276812 & $\begin{array}{l}\text { LOC297756/RPL34/RPS7/LOC306079/RPS26/SURF6/RPS27A/RGD156511 } \\
\text { 7/RGD1562381/RPS15/RGD1559808 }\end{array}$ \\
\hline CC & Autophagosome membrane & 0.003616 & 0.27785 & RAB7A/LAMP2/STX17/PRKD1 \\
\hline CC & Nuclear chromatin & 0.004689 & 0.308812 & $\begin{array}{l}\text { EP400/ARID1A/RBMXRTL/SFR1/HMGB2/RARA/CBX5/SMARCA5/MORF4L } \\
\text { 1/PSIP1/TCF3/RUNX2/MIXL1/ETV3/HIST2H4/HIST1H1A/MXD1/HIST1H1B }\end{array}$ \\
\hline CC & Autophagosome & 0.016393 & 0.669112 & FTH1/RAB7A/LAMP2/STX17/PRKD1/ATG12 \\
\hline CC & DNA packaging complex & 0.016574 & 0.669112 & HIST2H2BE/GLYR1/SMC4/TCF3/HIST2H4/HIST1H1A/HIST1H1B \\
\hline CC & Lateral plasma membrane & 0.016808 & 0.669112 & VANGL2/NSG1/PKD1/FGF13/GJB2 \\
\hline MF & Repressing transcription factor binding & 0.00545 & 0.759982 & CBX5/TCF3/RUNX2/RBPJ/MIXL1/ARNTL \\
\hline MF & Structural constituent of ribosome & 0.006363 & 0.759982 & $\begin{array}{l}\text { LOC297756/RPL34/RGD1564617/RPS7/RGD1562397/LOC306079/RPS26/ } \\
\text { RPS27A/RGD1565117/LOC100360679/RGD1562381/RPS15/MRPL3/ } \\
\text { RGD1559808 }\end{array}$ \\
\hline MF & $\begin{array}{l}\text { RNA polymerase II core promoter } \\
\text { proximal region sequence-specific DNA } \\
\text { binding }\end{array}$ & 0.00642 & 0.759982 & $\begin{array}{l}\text { NR4A3/LHX2/TCF3/NR3C1/ZFP384/ALS2CR8/MEIS2/RUNX2/GMEB2/SO } \\
\text { X6/RBPJ/HIVEP2/NKX6-1/MIXL1/PITX3/BCL11B/FOXS1/PAX1/MXD1/CRX }\end{array}$ \\
\hline MF & $\begin{array}{l}\text { Transcription factor activity, RNA } \\
\text { polymerase II core promoter proximal } \\
\text { region sequence-specific binding }\end{array}$ & 0.008708 & 0.759982 & $\begin{array}{l}\text { NR4A3/LHX2/TCF3/NR3C1/ZFP384/ALS2CR8/MEIS2/RUNX2/SOX6/RBPJ/ } \\
\text { HIVEP2/NKX6-1/MIXL1/PITX3/BCL11B/FOXS1/PAX1/MXD1/ARNTL/CRX }\end{array}$ \\
\hline MF & $\begin{array}{l}\text { Core promoter proximal region } \\
\text { sequence-specific DNA binding }\end{array}$ & 0.008925 & 0.759982 & $\begin{array}{l}\text { NR4A3/LHX2/TCF3/NR3C1/ZFP384/ALS2CR8/MEIS2/RUNX2/GMEB2/SO } \\
\text { X6/RBPJ/HIVEP2/NKX6-1/MIXL1/PITX3/BCL11B/FOXS1/PAX1/MXD1/CRX }\end{array}$ \\
\hline MF & $\begin{array}{l}\text { Core promoter proximal region DNA } \\
\text { binding }\end{array}$ & 0.009372 & 0.759982 & $\begin{array}{l}\text { NR4A3/LHX2/TCF3/NR3C1/ZFP384/ALS2CR8/MEIS2/RUNX2/GMEB2/SO } \\
\text { X6/RBPJ/HIVEP2/NKX6-1/MIXL1/PITX3/BCL11B/FOXS1/PAX1/MXD1/CRX }\end{array}$ \\
\hline MF & Manganese ion binding & 0.011064 & 0.759982 & GLUL/XPNPEP1/IMPA1/ARG1/TDP2 \\
\hline MF & $\begin{array}{l}\text { Transcriptional activator activity, RNA } \\
\text { polymerase II core promoter proximal } \\
\text { region sequence-specific binding }\end{array}$ & 0.011141 & 0.759982 & $\begin{array}{l}\text { NR4A3/LHX2/TCF3/NR3C1/ZFP384/ALS2CR8/MEIS2/RUNX2/RBPJ/HIVEP } \\
\text { 2/MIXL1/PITX3/BCL11B/PAX1/CRX }\end{array}$ \\
\hline MF & Histone binding & 0.011785 & 0.759982 & $\begin{array}{l}\text { CKS2/GLYR1/HMGB2/PHF1/CBX5/SMARCA5/USP15/ING1/ATAD2/HIST2 } \\
\text { H4/RAG2 }\end{array}$ \\
\hline MF & Methylated histone binding & 0.016167 & 0.759982 & GLYR1/PHF1/CBX5/ING1/RAG2 \\
\hline
\end{tabular}




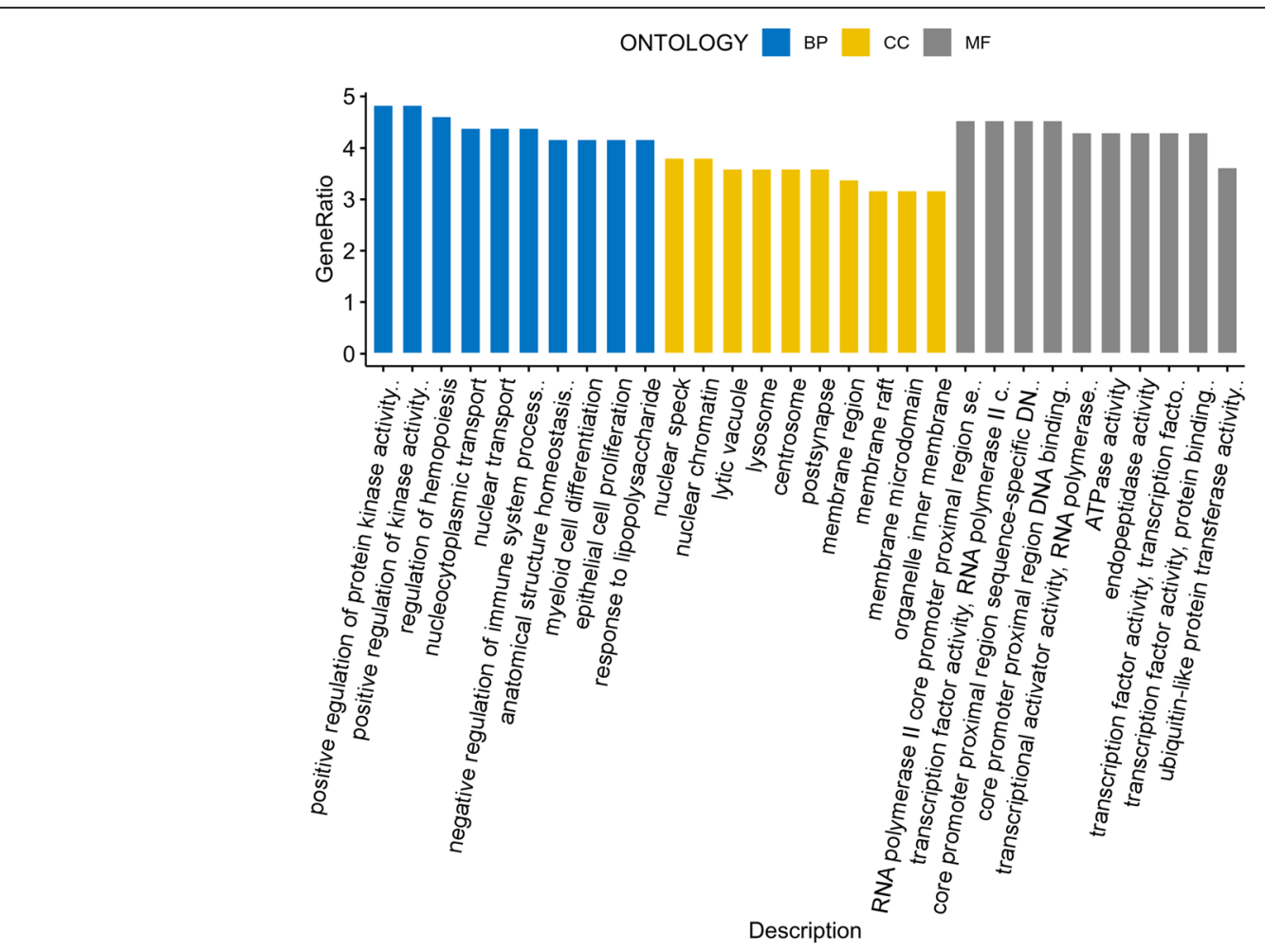

Fig. 4 Gene ontology analysis of differentially expressed genes in E-group and S-group

\section{Interaction network of DEGs and core genes in the interaction network}

Using data from the Cytoscape and STRING databases, the 10 hub nodes with the greatest degree of network connection were determined. The top 10 hub genes identified were RASL2-9, PSMD6, CPOX, FTH1, PYGL, GNAI1, PTPN1, RIC8A, RAB7A, LOC680441, USP4, and HIST2H2BE (Fig. 6 and Table 3). We listed top three MCODE results in Fig. 7.

\section{Discussion}

Subchondral bone remodeling is regulated by the bone resorption and bone formation, which mainly regulated by osteoclast and osteoblast respectively. Despite advancements in the understanding of the mechanism of OA, an effective method for accelerating the process remains to be identified. In this analysis, we found that 588 DEGs between E-group and S-group.

Bone remodeling is regulated by the balanced processes of osteoclast-mediated bone resorption and osteoblastmediated bone formation $[13,14]$. Disequilibrium of this balance leads to dysregulated bone tissue remodeling and can result in excessive bone loss or extra bone formation and consequent skeletal disease [15]. We identified DEGs that play roles in osteoclast and osteoblast differentiation and function in the early stages of OA in this model.

Table 4 KEGG pathway enrichment analysis of differentially expressed genes in E-group and S-group

\begin{tabular}{lllll}
\hline ID & Description & pvalue & p.adjust & gname \\
\hline rno00730 & Thiamine metabolism & 0.009585 & 0.866414 & ALPL/AKP3/AK1 \\
rno04923 & Regulation of lipolysis in adipocytes & 0.018613 & 0.866414 & GNAI3/PIK3CD/PLA2G16/AQP7/GNAS \\
rno05230 & Central carbon metabolism in cancer & 0.025683 & 0.866414 & PDHA1/HK1/PIK3CD/PFKP/ERBB2 \\
rno04915 & Estrogen signaling pathway & 0.026369 & 0.866414 & GNAI3/TFF1/PIK3CD/RARA/HSPA1B/ITPR1/GNAS/HSPA1L \\
rno04966 & Collecting duct acid secretion & 0.033955 & 0.866414 & ATP6V1G3/ATP6V1E1/CLCNKB \\
rno05162 & Measles & 0.039454 & 0.866414 & PIK3CD/IFIH1/EIF3H/RAB9A/FASLG/HSPA1B/CCND3/HSPA1L \\
rno00600 & Sphingolipid metabolism & 0.040658 & 0.866414 & B4GALT6/PPAP2B/ACER2/CERS1 \\
rno00983 & Drug metabolism-other enzymes & 0.042438 & 0.866414 & ITPA/CES2A/UGT2B5/GUSB/GSTA5/NAT2 \\
rno04710 & Circadian rhythm & 0.044488 & 0.866414 & PRKAB2/ARNTL/PER3 \\
\hline
\end{tabular}




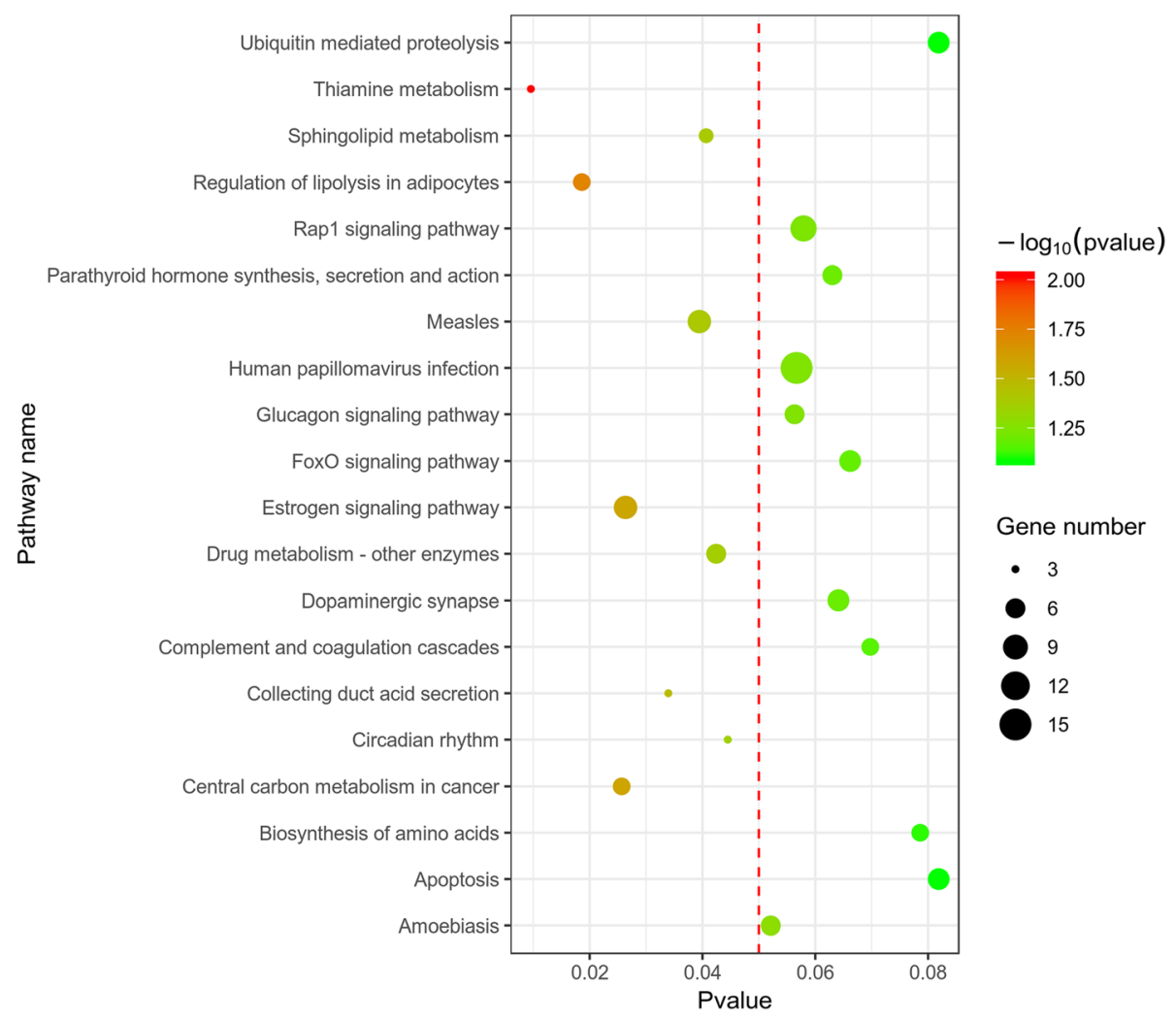

Fig. 5 The results of KEGG pathways enrichment analysis for DEGs based on clusterProfiler

Previous studies identified that an altered phenotype of subchondral osteoblasts and osteoclasts contribute to OA progress. Lamas et al. [16] reported that COL10A1 downregulation seems to have a role in the establishment of a defective and/or unstable subchondral cartilage matrix in OA disease. Ren et al. [17] used gene expression profile

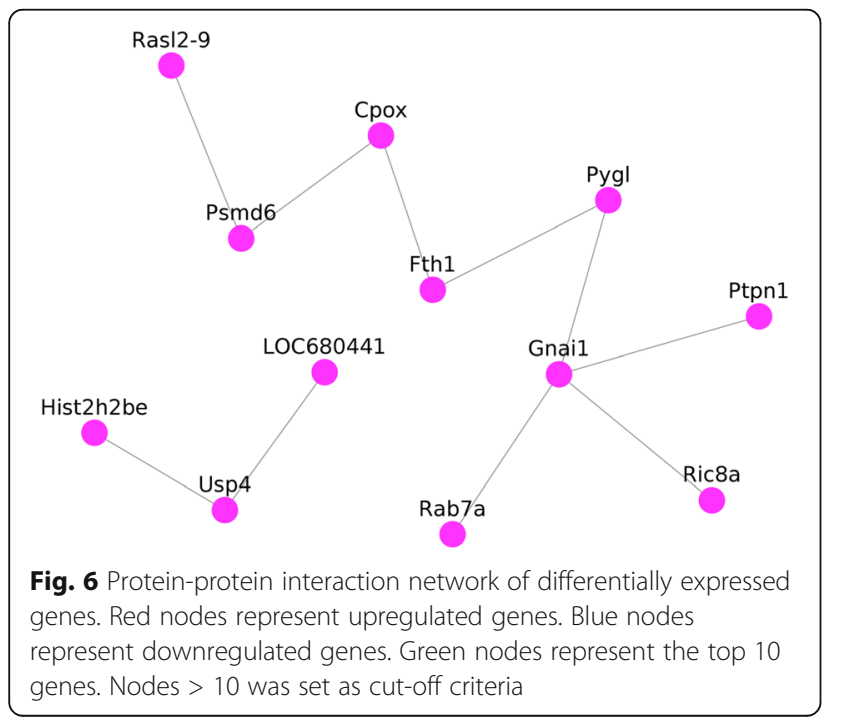

GSE103416 to identify the different expression genes and potential pathways. Results show that Gna13/cGMP-PKG signaling pathway was identified as a potential research target for therapy and for further understanding the development of OA.

We further performed Kegg pathways to identify the potential pathways that involving in the progress of OA. We found that Rap1 signaling pathway was the most obvious different signaling pathways. For Rap1 signaling pathway, we found nine DEGs (GNAI3/RAPGEF5/CSF1/PIK3CD/ MK1/LPAR2/P2RY1/GNAS/PRKD1/RASSF5). Another potential pathway was estrogen signaling pathway. Ren et al. [17] used gene expression profile GSE103416 and found that Gna13/cGMP-PKG signaling pathway was identified as a potential research target for therapy and for further understanding the development of OA. Feng et al. [18] revealed that PDGFRB, IFNG, EGR1, FASLG, and H3F3B may be the potential targets for OA diagnosis and treatment. Liang Y al [19]. found that estrogen deficiency is closely related to the development of menopausal arthritis including OA. Estrogen acts via ER and miR-140 to inhibit the catabolic activity of proteases within the chondrocyte extracellular matrix.

We also found that Rap1 signaling pathway participated into the pathological process of OA. Zhang et al. [20] 

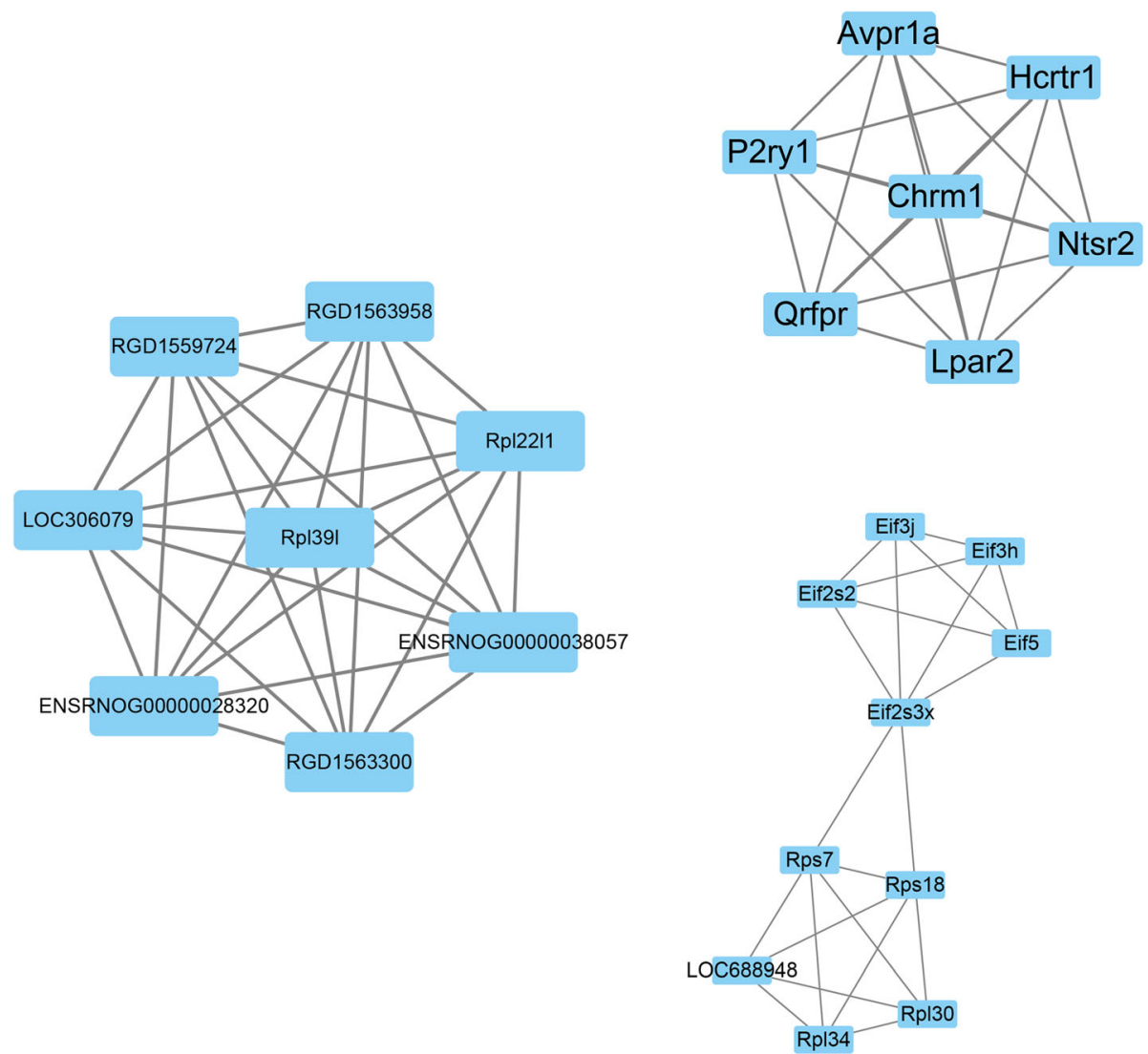

Fig. 7 The top 3 modules from the gene-gene interaction network. The squares represent the differentially expressed genes (DEGs) in modules, and the lines show the interaction between the DEGs

performed a gene expression analyses of subchondral bone in early experimental osteoarthritis by microarray. Results found that Alp, Igf1, Tgf $\beta 1$, Postn, Mmp3, Tnfsf11, Acp5, Bmp5, Aspn, and Ihh genes that involved in the pathological process of $\mathrm{OA}$, and they also performed PCR to identify these DEGs in the OA and normal cartilage. Kovács et al. [21] also revealed that the Wnt and the OPG-RANKL-RANK signaling systems, as key mediators, interact in subchondral bone remodeling in OA development, which indicated that subchondral bone remodeling also affects OA. Zhou et al. [22] revealed that matrix metalloproteinase (MMP)1, MMP3, MMP13, and prostaglandin-endoperoxide synthase 2 (PTGS2) are associated with human developmental chondrogenesis.

\section{Conclusion}

In summary, totally, 588 DEGs were identified including 199 upregulated DEGs and 389 downregulated DEGs screened in OA and sham-operation. We identified 12 core genes, including RASL2-9, PSMD6, CPOX, FTH1, PYGL, GNAI1, PTPN1, RIC8A, RAB7A, LOC680441, USP4, and HIST2H2BE. Additional experimental studies will be needed to validate our findings.

\section{Abbreviations}

OA: Osteoarthritis; DAVID: Database for Annotation, Visualization and Integrated Discovery; DEGs: Differentially expressed genes; GO: Gene ontology; KEGG: Kyoto Encyclopedia of Genes and Genomes; PPI: Proteinprotein interaction; GEO: Gene Expression Omnibus; FC: Fold change; BP: Biological process; MF: Molecular function; CC: Cellular component; STRI NG: Search Tool for the Retrieval of Interacting Genes; MCODE: Molecular Complex Detection

\section{Acknowledgements}

None.

\section{Authors' contributions}

ZW designed the study and developed the retrieve strategy. HWB and JY searched and screened the summaries and titles. ZW. HWB, and JY drafted the article. All authors read and approved the final draft.

Funding

Not applicable.

Availability of data and materials

The data was freely downloaded from the public GEO database.

Ethics approval and consent to participate

Not applicable. This paper does not involve research on humans.

Consent for publication

Not applicable. 


\section{Competing interests}

The authors declare that they have no competing interests.

\section{Author details}

'Department of Orthopaedics, Jingjiang People's Hospital, No. 28, Zhongzhou Road, Jingjiang, Taizhou 214500, Jiangsu Province, China. ${ }^{2}$ Department of General Surgery, Jingjiang People's Hospital, No. 28, Zhongzhou Road, Jingjiang, Taizhou 214500, Jiangsu Province, China.

Received: 6 November 2019 Accepted: 30 July 2020

Published online: 08 August 2020

\section{References}

1. Lories RJ, Luyten FP. The bone-cartilage unit in osteoarthritis. Nat Rev Rheumatol. 2011;7:43-9. https://doi.org/10.1038/nrrheum.2010.197.

2. Moskowitz RW. The burden of osteoarthritis: clinical and quality-of-life issues. Am J Manag Care. 2009;15:S223-9.

3. Madry H, van Dijk CN, Mueller-Gerbl M. The basic science of the subchondral bone. Knee Surg Sports Traumatol Arthrosc. 2010;18:419-33. https://doi.org/10.1007/s00167-010-1054-z.

4. Bobinac D, Marinovic M, Bazdulj E, et al. Microstructural alterations of femoral head articular cartilage and subchondral bone in osteoarthritis and osteoporosis. Osteoarthr Cartil. 2013;21:1724-30. https://doi.org/10.1016/j. joca.2013.06.030.

5. Razmara E, Bitaraf A, Yousefi $\mathrm{H}$, et al. Non-Coding RNAs in Cartilage Development: An Updated Review. Int J Mol Sci. 2019;20. https://doi.org/10. 3390/ijms20184475.

6. Elgaaen BV, Olstad OK, Sandvik L, et al. ZNF385B and VEGFA are strongly differentially expressed in serous ovarian carcinomas and correlate with survival. PLoS ONE. 2012;7:e46317. https://doi.org/10.1371/journal.pone. 0046317.

7. Al-Rekabi Z, Wheeler MM, Leonard A, et al. Activation of the IGF1 pathway mediates changes in cellular contractility and motility in single-suture craniosynostosis. J Cell Sci. 2016;129:483-91. https://doi.org/10.1242/jcs. 175976

8. Gene Ontology Consortium. The Gene Ontology (GO) project in 2006. Nucleic Acids Res. 2006:34:D322-6. https://doi.org/10.1093/nar/gkj021.

9. Ashburner M, Ball CA, Blake JA, et al. Gene ontology: tool for the unification of biology. The Gene Ontology Consortium. Nat Genet. 2000;25:25-9. https://doi.org/10.1038/75556.

10. Kanehisa M, Goto S. KEGG: kyoto encyclopedia of genes and genomes. Nucleic Acids Res. 2000;28:27-30. https://doi.org/10.1093/nar/28.1.27.

11. Szklarczyk D, Franceschini A, Wyder S, et al. STRING v10: protein-protein interaction networks, integrated over the tree of life. Nucleic Acids Res. 2015;43:D447-52. https://doi.org/10.1093/nar/gku1003.

12. Bandettini WP, Kellman P, Mancini C, et al. MultiContrast Delayed Enhancement (MCODE) improves detection of subendocardial myocardial infarction by late gadolinium enhancement cardiovascular magnetic resonance: a clinical validation study. J Cardiovasc Magn Reson. 2012;14:83. https://doi.org/10.1186/1532-429x-14-83.

13. Li G, Yin J, Gao J, et al. Subchondral bone in osteoarthritis: insight into risk factors and microstructural changes. Arthritis Res Ther. 2013;15:223. https:// doi.org/10.1186/ar4405.

14. Aizah N, Chong PP, Kamarul T. Early Alterations of Subchondral Bone in the Rat Anterior Cruciate Ligament Transection Model of Osteoarthritis. Cartilage. 2019;1947603519878479. https://doi.org/10.1177/ 1947603519878479.

15. Martig S, Hitchens PL, Lee PVS, et al. The relationship between microstructure, stiffness and compressive fatigue life of equine subchondral bone. J Mech Behav Biomed Mater. 2019;101:103439. https://doi.org/10. 1016/j.jmbbm.2019.103439.

16. Lamas JR, Rodriguez-Rodriguez L, Vigo AG, et al. Large-scale gene expression in bone marrow mesenchymal stem cells: a putative role for COL10A1 in osteoarthritis. Ann Rheum Dis. 2010;69:1880-5. https://doi.org/ 10.1136/ard.2009.122564

17. Ren YM, Zhao X. Exploring the key genes and pathways of osteoarthritis in knee cartilage in a rat model using gene expression profiling. Yonsei Med J. 2018;59:760-8. https://doi.org/10.3349/ymj.2018.59.6.760.

18. Feng Z, Lian KJ. Identification of genes and pathways associated with osteoarthritis by bioinformatics analyses. Eur Rev Med Pharmacol Sci. 2015; 19:736-44.
19. Liang $Y$, Duan $L$, Xiong J, et al. E2 regulates MMP-13 via targeting miR-140 in IL-1 beta-induced extracellular matrix degradation in human chondrocytes. Arthritis Res Ther. 2016;18:105. https://doi.org/10.1186/ s13075-016-0997-y.

20. Zhang R, Fang $H$, Chen $Y$, et al. Gene expression analyses of subchondral bone in early experimental osteoarthritis by microarray. PLoS ONE. 2012;7: e32356. https://doi.org/10.1371/journal.pone.0032356.

21. Kovacs B, Vajda E, Nagy EE. Regulatory effects and interactions of the Wnt and OPG-RANKL-RANK signaling at the bone-cartilage interface in osteoarthritis. Int J Mol Sci. 2019;20. https://doi.org/10.3390/ijms20184653.

22. Zhou J, Li C, Yu A, et al. Bioinformatics analysis of differentially expressed genes involved in human developmental chondrogenesis. Medicine (Baltimore). 2019;98:e16240. https://doi.org/10.1097/md.0000000000016240.

\section{Publisher's Note}

Springer Nature remains neutral with regard to jurisdictional claims in published maps and institutional affiliations.

\section{Ready to submit your research? Choose BMC and benefit from:}

- fast, convenient online submission

- thorough peer review by experienced researchers in your field

- rapid publication on acceptance

- support for research data, including large and complex data types

- gold Open Access which fosters wider collaboration and increased citations

- maximum visibility for your research: over $100 \mathrm{M}$ website views per year

At BMC, research is always in progress.

Learn more biomedcentral.com/submissions 